\title{
Constraints on the optical-IR extragalactic background from $\gamma$-ray absorption studies
}

\author{
Luigi Costamante ${ }^{1,2}$ \\ ${ }^{1}$ Stanford University, Stanford, USA \\ ${ }^{2}$ INAF, Brera Observatory, Milano, Italy \\ email: luigic2011@gmail.com
}

\begin{abstract}
Very high energy (VHE $\gtrsim 0.1 \mathrm{TeV}$ ) gamma-rays from extragalactic sources, interacting by $\gamma-\gamma$ collisions with diffuse intergalactic radiation fields, provide an alternative way to constrain the diffuse background light, completely independent of direct measurements. The limits depend however on our knowledge of the physics of the gamma-ray sources. After clarifying the interplay between background light and VHE spectra, I summarize the extent and validity of the obtainable limits, and where future improvements can be expected.
\end{abstract}

Keywords. galaxies: active, BL Lacertae objects: general, cosmology: diffuse radiation, gamma rays: observations, infrared: galaxies.

\section{Introduction}

VHE $\gamma$-rays $\dagger$ from extragalactic sources provide an alternative and completely independent way, with respect to direct measurements, for probing the diffuse Extragalactic Background Light (EBL, meaning from UV to far infrared wavelengths; e.g. Hauser \& Dwek 2001). This approach is based on the study of "absorption" features imprinted on the $\mathrm{GeV}-\mathrm{TeV}$ spectra by the interaction with EBL photons through the pair-creation process $\left(\gamma \gamma \rightarrow e^{+} e^{-}\right.$, see e.g. Aharonian 2001 and references therein). Blazars represent a very useful class of $\gamma$-ray beamers, being numerous over a wide range of redshifts, very luminous (enhanced by relativistic beaming) and long-lasting sources. However, they are far from being standard candles, and therefore the constraints on the EBL that can be derived are always heavily dependent on our understanding of the blazar intrinsic emission and physical properties.

Nevertheless, it is possible to derive meaningful constraints if the intrinsic blazar properties implied by certain EBL spectra are very far from the observed or expected range, and/or become inconsistent with other features in a blazar's spectral energy distribution (SED). The observations of the last 5 years have allowed substantial progress, and provided the strongest constraints to date. Here both the main results and the limits of their validity are discussed.

\section{Diagnostics: how absorption deforms $\mathrm{TeV}$ spectra}

The convolution of the $\gamma-\gamma$ cross section with the EBL spectrum yields an energydependent attenuation. Because the optical depth $\tau$ mostly increases with $\gamma$-ray energy, the emerging $\gamma$-ray spectrum is observed steeper than the initial, intrinsic spectrum

$\dagger$ In the following I will use 'VHE' and 'TeV'as interchangeable terms, meaning \pm 1 decade around $1 \mathrm{TeV}$. The same for 'HE' (high-energy, $>0.1 \mathrm{GeV}$ ) and 'GeV'. Considering where detectors have the best sensitivity and resolution for typical astronomical spectra, Cherenkov telescopes are in fact $\mathrm{TeV}$ instruments and the Fermi-LAT detector is a $\mathrm{GeV}$ instrument. 

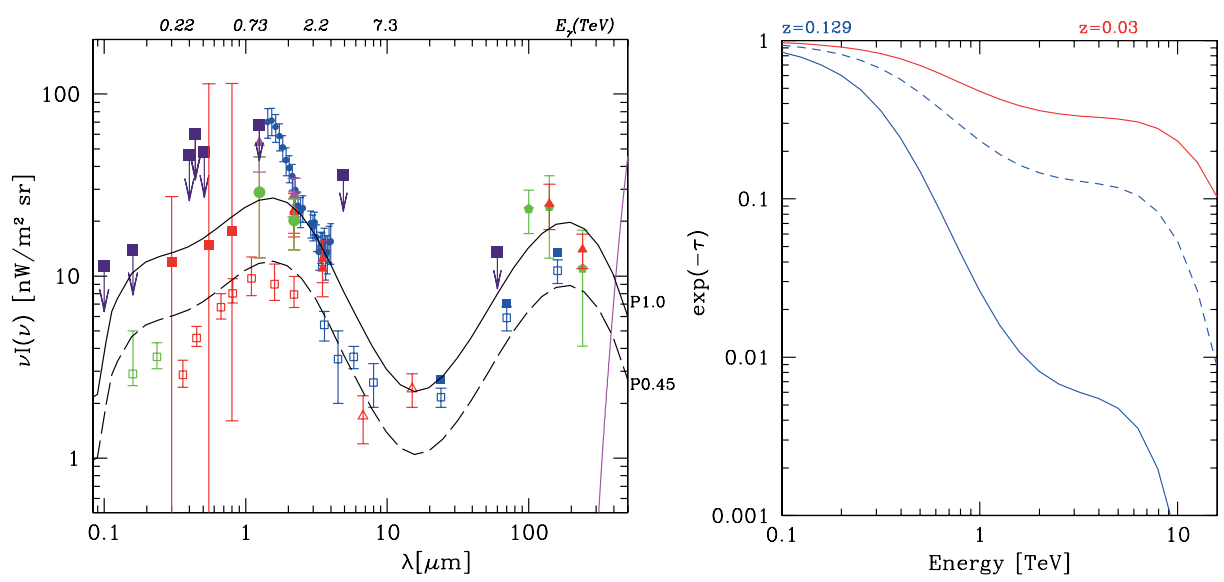

Figure 1. Left: SED of the EBL, as was discussed in the year 2005 (from Aharonian et al. 2006). Open symbols show lower limits from galaxy counts, while filled symbols correspond to direct estimates. The annotations on the upper axis denote the VHE energy corresponding to the peak of the $\gamma-\gamma$ cross section. The two curves (identical except for normalization) are plotted as simple shapes reproducing the main EBL features. Right: the attenuation curves $e^{-\tau}$ corresponding to the EBL curves in the left panel (lower solid and dashed lines), and at two different redshifts for the upper EBL curve (lower and upper solid lines). The attenuation curves represent directly the shape of an observed VHE spectrum, if the initial spectrum were a power-law parallel to the upper axis. All calculations performed assuming $H_{0}=70 \mathrm{~km} / \mathrm{s} / \mathrm{Mpc}$, $\Omega_{m}=0.3, \Omega_{\Lambda}=0.7$.

emitted by the source (namely, the photon index $\Gamma_{o b s} \geqslant \Gamma_{i n t}$ ). This can be seen in Fig. 1, where the attenuation factor $e^{-\tau}$ is plotted as a function of energy. Limits on the EBL can be thus derived if the intrinsic spectrum required by a particular EBL intensity or shape is too anomalous (typically too hard) with respect to the known physics of the source (Costamante et al. 2004). Several points can be noted from Fig. 1:

1) different EBL wavebands (having different EBL spectra) affect each part of the $\gamma$-ray spectrum in a different way, yielding a complex deformation shape. However, over some ranges (namely $0.2-1 \mathrm{TeV}$ and $2-8 \mathrm{TeV}$ ), it approximates a power-law shape. That is, if the intrinsic spectrum is a power-law, the observed spectrum can be well fitted by a power-law of steeper index.

2) The amount of steepening $\Delta \Gamma$ increases both with distance and EBL intensity. Thus redshift gives leverage: the same difference in EBL flux $\Delta F_{\mathrm{EBL}}$ causes a higher $\Delta \Gamma$ at larger distances. More distant sources provide therefore more sensitivity for EBL constraints, but at the same time they suffer stronger attenuation, yielding much lower statistics. As a result, there is an optimal range of redshifts that provides the best compromise between sensitivity and statistical uncertainty, and thus gives the most stringent EBL limits. With the present detectors, it is around $z \sim 0.15-0.25$.

3) If the EBL number density $n(\epsilon) \propto \epsilon^{-\beta}$ (i.e. the EBL SED $\propto \lambda^{\beta-2}$ ), the optical depth $\tau\left(E_{\gamma}\right)$ becomes $\propto E_{\gamma}^{\beta-1}$. Where $\beta \approx 1$, the optical depth thus becomes independent of energy (Aharonian 2001). In such case there is no steepening: the absorbed spectrum reproduces the original shape, simply attenuated by a constant factor. This is what partly happens in the EBL waveband 3-10 $\mu \mathrm{m}$, according to all most recent EBL calculations (e.g. Franceschini et al. 2008, Dominguez et al. 2011, Gilmore et al. 2011, Kneiske \& Dole 2010). It yields a flattening feature in the attenuation curve between $\sim 1-2$ and $8-9$ 
$\mathrm{TeV}$. There, the observed $\gamma$-ray spectrum partly recovers its intrinsic slope and thus can appear harder than in the $0.2-1 \mathrm{TeV}$ band $\dagger$

4) Therefore, NO cutoff is produced by EBL absorption between 0.2 and $8 \mathrm{TeV}$ ! Any cutoff or steepening seen in this energy range is most likely intrinsic to the source spectrum. Fig. 1 also clarifies why almost all "data points" reported on the so-called "gammaray horizon" plots in the literature (showing the $E_{\gamma}$ at which $\tau=1$ as a function of redshift) are basically meaningless: there is no way to measure an EBL cutoff between 0.1 and $10 \mathrm{TeV}$ using only VHE data. The only two cutoffs from EBL are between the HE and VHE bands, i.e. between 20 and $200 \mathrm{GeV}$ (thus requiring a Fermi-LAT spectrum), or above 8-10 TeV (for which only two sources have been detected with sufficient statistics so far).

5) The $\gamma$-ray steepening is determined by the difference in optical depth between the two ends of an observed VHE band. Therefore any spectral steepening (and consequently any EBL upper limit) can always be counteracted and cancelled by an appropriate increase of the optical depth (i.e. of the EBL flux) at the lower $\gamma$-ray energies, so as to equalize the optical depths. In the $0.1-1 \mathrm{TeV}$ range, this is obtained by increasing the UV flux with respect to the NIR flux (see e.g Aharonian et al. 2006). Therefore, any limit on the EBL depends always on an assumption on the $U V$ flux, by definition. All claims of "new" methods for "model-independent" or "shape-independent" EBL limits (e.g. Mazin \& Raue 2007, Hinton \& Hofmann 2009) are therefore essentially incorrect, even if their (hidden) assumptions are reasonable and in fact commonly used (e.g. assuming that the UV flux is always lower than the optical-NIR flux).

\section{Optical-NIR constraints}

Until 2005, the large uncertainty especially around $0.8-3 \mu \mathrm{m}$ (a factor $5-10 \times$ between lower limits and direct estimates) caused a fundamental ambiguity in the interpretation of the gamma-ray spectra of blazars. An observed TeV spectrum could be the result either of a hard intrinsic spectrum attenuated by a high-density EBL, or of a soft intrinsic spectrum less absorbed by a low-density EBL. In both cases, the required intrinsic VHE spectrum was well within the typical range shown so far by blazars, so no useful constraint could be derived (with the exception of the $2-10 \mu \mathrm{m}$ range, see Aharonian et al. 2003). This ambiguity essentially undermined our capability to study blazars, since it corresponded to large differences both in the energy and luminosity of the $\gamma$-ray peak in the SED.

In 2005, however, the HESS observations of the BL Lac objects 1ES 1101-232 ( $z=0.186)$ and H 2356-309 ( $z=0.165$ ) provided a fundamental breakthrough (Aharonian et al. 2006). The observed $\gamma$-ray spectra (detected between 0.2 and 1-3 TeV) were much harder than expected for their redshift, implying extremely hard intrinsic spectra $\left(\Gamma_{\text {int }} \lesssim 0\right)$ for high EBL densities (Fig. 2). Such hard spectra were never seen in the closer, less absorbed objects and were at odds with all the known blazar physics and phenomenology. They were also not supported by same-epoch multiwavelength observations of their SED. A low EBL intensity could instead accommodate all the new data within the typical range of blazar properties. Assuming that the intrinsic spectra were not harder than $\Gamma=1.5$ an upper limit could be derived, which resulted in limts on the EBL very close to the lower limits given by the integrated light of known galaxies (details in Aharonian et al. 2006).

$\dagger$ In fact, $\gamma-\gamma$ absorption can make a gamma-ray spectrum even harder than originally emitted, when $n(\epsilon) \propto \epsilon^{>-1}$ (e.g. for a Planckian distribution). This is indeed an effective way to explain very hard $\gamma$-ray spectra (Aharonian et al. 2008). 

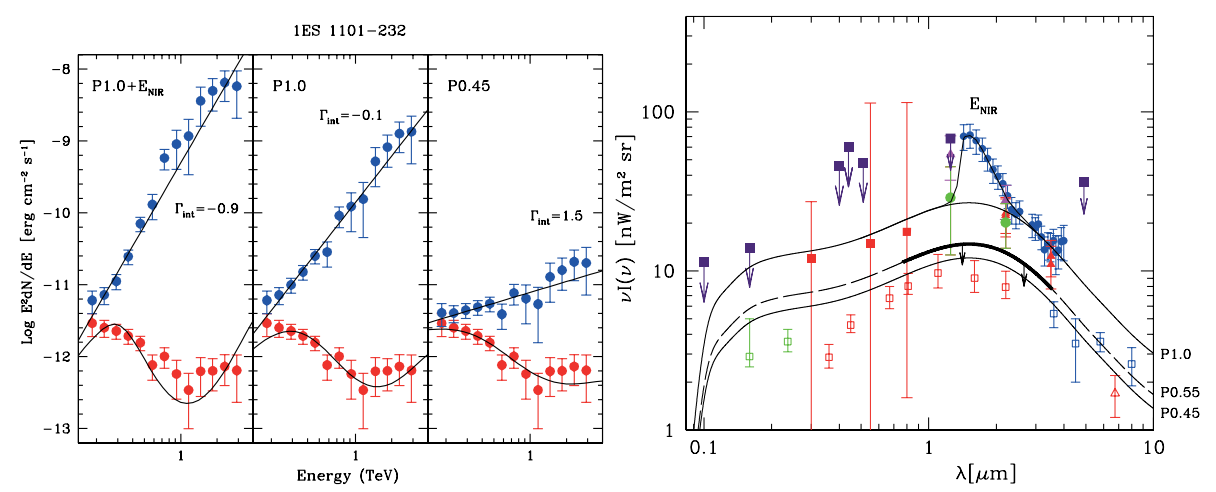

Figure 2. Left: HESS spectra of 1ES 1101-232, corrected for absorption with three different EBL SEDs, as labelled (from Aharonian et al. 2006). Lower points: observed data. Upper points: absorption-corrected data. The lines show the best fit power-laws to the reconstructed spectrum, and the corresponding shape after absorption. Right: upper limit for the EBL (black shaded region), derived assuming a blazar spectrum with $\Gamma \geqslant 1.5$.

This result is now further corroborated by several other sources and observations (e.g. Aharonian et al. 2007a , Acciari et al. 2009, Albert et al. 2009).

This result has three main consequences: 1) it pins down the origin of the EBL, showing that at these wavelengths it is strongly dominated by the direct starlight from galaxies, excluding a strong contribution from other sources like Pop-III stars (Santos et al. 2002); 2 ) it means that the intergalactic space is more transparent to $\gamma$-rays than previously thought, thus enlarging the $\gamma$-ray horizon; 3 ) it strongly reduces the ambiguity on $\mathrm{TeV}$ spectra of blazars.

\subsection{The (in)famous limit of $\Gamma=1.5$.}

There is a lot of confusion in literature on the validity of the $\Gamma=1.5$ hardness limit and on the reasons for its adoption. It is important to understand that this is NOT the hardest possible theoretical spectrum in blazars, and was never introduced as such (see Aharonian et al. 2006). Indeed there are many possible mechanisms to produce extremely hard gamma-ray spectra in blazars, such as bulk-motion comptonization (Aharonian 2001), internal absoption on narrow-band photon fields (Aharonian et al. 2008), uncooled particle acceleration spectra or fine-tuned shock acceleration (yielding however $\Gamma \sim 1.2$, e.g. Stecker et al. 2007), a low-energy cutoff in the particle distribution at very high energy (e.g. Katarzynski et al. 2006, Lefa et al. 2011), or relativistic Maxwellian distributions (e.g. Saugé \& Henri 2004, Lefa et al. 2011).

The $\Gamma=1.5$ limit is a reference value, a benchmark that so far marks the borderline between reality and speculation. Observations do show that blazars can have photon spectra with $\Gamma \gtrsim 1.5$ (directly seen in synchrotron or inverse Compton emissions), and these can be produced with standard shock acceleration and cooling mechanisms without invoking special conditions or fine-tuning. On the other hand, much harder spectra have never been significantly detected in blazars so far at high particle energies, neither directly or by synchrotron emission. The alternative scenarios, though not excluded by observations, are not suggested by them either, and in some cases require extreme fine-tuning to avoid conflicts with multiwavelength SED data.

Furthermore, the $\Gamma=1.5$ value should not be considered a sharp, hard limit. At any given redshift, EBL absorption establishes a one-to-one relation between the observed and intrinsic $\gamma$-ray spectral slope. Thus a different assumption shifts the EBL limit accordingly, but note that only a large change (e.g. $\Gamma_{\text {int }}<<1$ ) can effectively impact the 

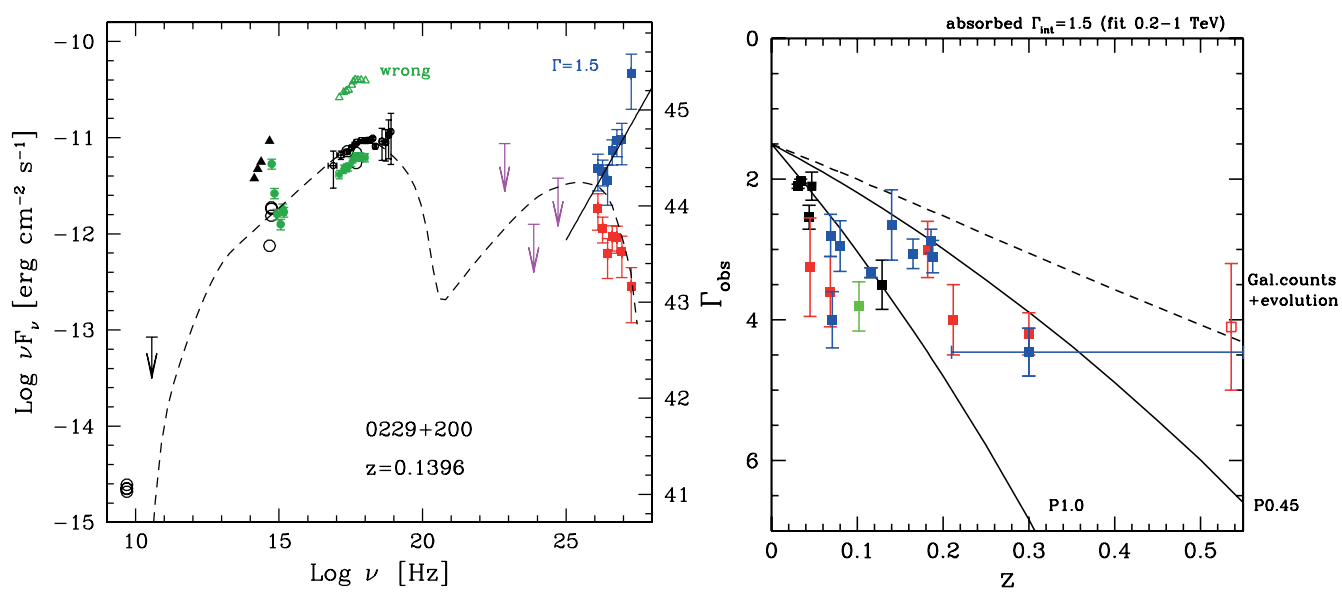

Figure 3. Left: the SED of $1 \mathrm{ES} 0229+200$, showing the correct X-ray data from Swift (filled circles), at much lower flux than the wrong X-ray data (upper open triangles) used in Tavecchio et al. 2009. Right: observed VHE photon indices of all detected TeV blazars as a function of redshift. The lines represent the expected observed (i.e. absorbed) photon index of a source with an intrinsic spectrum $\Gamma=1.5$, when fitted in the range $0.2-1 \mathrm{TeV}$, for different EBL levels (as labelled). The separation of the data from the lines at each redshift shows how much the intrinsic spectrum is harder or softer than 1.5. Adapted from Costamante 2006.

conclusion of a low EBL. For example, in the case of 1ES 1101-232, a shift of $\Delta \Gamma=0.3$ (e.g. by assuming $\Gamma_{\text {int }} \sim 1.2$ ) affects the EBL limit by no more than $10 \%$ (Aharonian et al. 2006). The optical-NIR upper limit is therefore robust to such changes in blazar assumptions, which are of the same order of the statistical and systematic uncertainties in the $\gamma$-ray spectra.

The only relevant issue left, therefore, is to assess - theoretically and observationally if blazars do have emission components characterized by extremely hard spectra (e.g. $\Gamma=0.7$ or less) or not, and how they behave.

\section{Why a low EBL density is still the most likely solution.}

Despite the several possible scenarios for producing very hard spectra in blazars, there are two main reasons why a low EBL density seems still the correct solution.

The first concerns the spectrum of the emitting particles. Most of these scenarios invoke very hard particle spectra, either as a pile-up/Maxwellian distribution or as a low-energy cutoff, and suppressing radiative cooling. These mechanisms look feasable even in a one-zone synchrotron self-Compton scenario for blazars (see Lefa et al. 2011 for a recent comprehensive discussion). However, since the synchrotron emission (which in these objects corresponds to optical-X-ray energies) traces directly the particle spectrum, such hard features should appear in the synchrotron spectrum as well, at some energies and at least in some epochs. Instead, they have never been observed in more than 30 years of X-rays and optical/IR observations of blazars, requiring a "cosmic conspiracy" to always hide the hard emission below a more normal component or in seldom-observed bands $(\mathrm{mm})$. Intriguingly, there was a recent claim of possible observational evidence for a low-energy cutoff in the Swift data of 1ES 0229+200, as an unusually high X-ray-toUV flux ratio Tavecchio et al. 2009. However, a bug was found in the calculation of the effective area of the X-ray data, and the correct result (see Fig. 3) show a spectrum in line with all other objects and observations. Future observations with ALMA (mm) and 

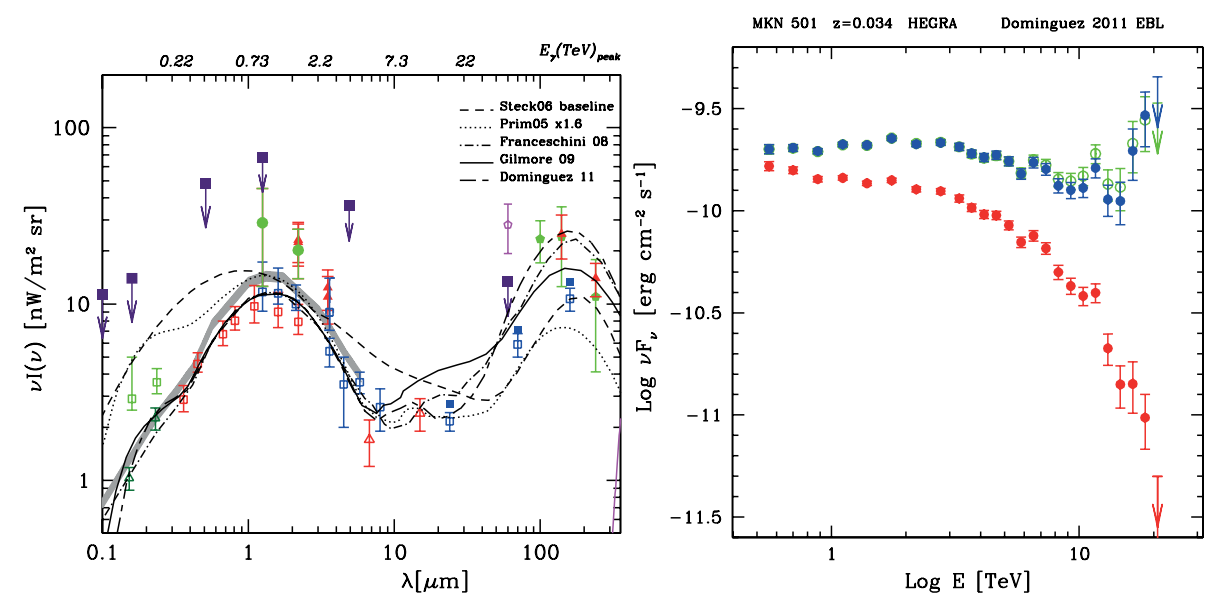

Figure 4. Left: SED of the EBL with the most recent models. The grey band shows the EBL upper limits in the Opt-NIR range (with the $+1 \sigma$ statistical uncertainty from the VHE data) recalculated using the EBL shape by Franceschini et al. (2008). Right: HEGRA spectrum of Mkn 501, as observed (red, lower points) and absorption-corrected with two recent EBL-model calculations (Dominguez et al. 2011 and Gilmore et al. 2011, upper black-blue filled and grey-green open points, respectively). Both cases show an upturn.

NuSTAR (hard X-ray) may provide further insights. Without invoking a hard particle spectrum, internal absorption on a narrow-banded photon field can make the gamma-ray spectrum extremely hard (Aharonian et al. 2008) However, large fluxes in the GeV range are required, and these seem now excluded by the low fluxes or non-detection of the hard-TeV sources with Fermi-LAT (Abdo et al. 2010).

The second reason for a low EBL density (and thus for $\Gamma_{i n t} \gtrsim 1.5$ ) is that a high EBL would require a dramatic change of blazar properties in a very narrow range of redshifts (see Fig. 3). A high EBL would create a sharp dicotomy in the spectra of blazars around $z=0.15$ : all sources below this redshift would have $\Gamma \geqslant 1.5$, while all blazars above this redshift would always have $\Gamma<1.5$, all other properties being equal or very similar. There is no known reason, observational bias or cosmic physical parameter that can explain such an abrupt change of properties over such a small redshift range. Instead, a low EBL intensity makes the range of $\mathrm{TeV}$ blazar spectra consistent among each other for all redshifts sampled so far.

Though not yet "bullet-proof", until proven otherwise a low EBL seems the preferable solution according to Occam's razor. (see also Madau \& Silk 2005 for the cosmological problems to explain a high EBL flux).

\section{The Chain of Constraints}

Since the EBL photon field is the same for all sources, and evolving in redshift, the hardness limits on the $\gamma$-ray spectrum from different blazars and in different energy bands can be combined to constrain the EBL over a wide range of wavelengths, and in a more stringent way than allowed by each single object (Costamante et al. 2004, Dwek \& Krennrich 2005, Mazin \& Raue 2007). Each new spectrum can take advantage of the previous limits, forming a chain of constraints that starts with (and depends on) the assumption on the UV flux. With the reasonable hypothesis that the UV background is lower than the Opt-NIR one (e.g. around 2-6 $\mathrm{nW} / \mathrm{m}^{2} \mathrm{sr}$ ), the blazar spectra between 0.1 
and $1 \mathrm{TeV}$ pin down the Opt-NIR flux very close to the galaxy counts limit (Aharonian et al. 2006).

With this limit, the blazar spectra between $\sim 1$ and $10 \mathrm{TeV}(1 \mathrm{ES} 0229+200$; Aharonian et al. 2007b and 1ES 1426+428; Aharonian et al. 2003) constrain the EBL spectrum between 2 and $10 \mu \mathrm{m}$ to a slope close to $\lambda^{-1}$, again very close to the lower limits from galaxy counts (Aharonian et al. 2007b). This is possible because the Opt-NIR limit precludes the possibility of increasing the $\sim 1 \mu \mathrm{m}$ EBL flux to reduce the difference in optical depth between 1 and $10 \mathrm{TeV}$ (see Sect. 2).

The consequent upper limit at $10 \mu \mathrm{m}$ constrains the rising of the EBL SED towards the far-infrared hump, which is determined by warm and cold dust emission. This band is probed by $\gamma$-rays between $\sim 8$ and $100 \mathrm{TeV}$. So far, only two close-by objects have been measured up to 15-20 TeV, thanks to HEGRA observations: Mkn 421 ( $z=0.031$; Aharonian et al. 1999) and Mkn 501 ( $z=0.034$; Aharonian et al. 2001). Interestingly, the HEGRA spectrum of Mkn 501 seems to have problems with the most recent EBL calculations, which cause an up-turn or pile-up at the highest energies (see Fig. 4). This is the modern version of the so called "TeV-FIR background crisis" (see e.g. Aharonian 2001), caused by a first very high estimate of the $50 \mu \mathrm{m}$ EBL flux. However the information on warm dust and the $\gamma$-ray statistic are still insufficient to draw any sensible conclusion. More data in the $>10 \mathrm{TeV}$ range are needed (hopefully from CTA).

\section{Future and Conclusions}

VHE data sample mainly the local EBL $(z=0-0.5)$. To probe the EBL evolution over cosmic time and in the UV region, sources up to $z=3-4$ and data in the 10-100 $\mathrm{GeV}$ range are needed. The first results from Fermi-LAT (Abdo et al. 2010b) are in agreement with the VHE limits and with the most recent EBL evolution models. In this respect, however, GRBs might turn out to be more useful $\gamma$-ray sources, given that EBL and blazar-intrinsic evolutionary effects might be very difficult to disentangle (see e.g. Reimer 2007).

At VHE, on te other hand, CTA will NOT improve the Opt-NIR EBL per se, contrary to various claims. The reason is twofold. Firstly, there is not much room for improvement! The present upper limits already match the lower limits by galaxy counts quite closely (see Fig. 4). Secondly, even with infinite $\gamma$-ray statistics, there remains the unavoidable systematic uncertainty in blazar modelling. The small $\Delta \Gamma \sim 0.1-0.3$ induced by the residual EBL uncertainty between lower and upper limits can be typically accommodated with very small changes in blazar parameters, for a given set of multiwavelength data.

The main question to be addressed is whether our blazar assumptions are correct (in which case the EBL flux is already pinned down) or are not (and by how much). In the latter case, the entire construction must be revisited, since our understanding of the gamma-ray sources would have changed completely. It is on this aspect, on blazar physics, that CTA is expected to provide the most significant improvements and to test our assumptions by finding counter examples. This can be done by monitoring more low-redshift objects in the $\mathrm{TeV}$ range (to detect directly $\Gamma<1$ ) and by measuring more high-redshift spectra (to find those for which $\Gamma=1.5$ is incompatible with galaxy counts; Costamante 2007).

In conclusion, a low EBL close to galaxy counts seems the "convergent solution", despite some uncertainties in blazar physics. However, there are still some fundamental aspects of the acceleration and emission mechanisms in blazars that are not yet understood, which present/future observations are/will test. The EBL limits obtained so far 
are robust against small changes in assumptions about blazars, but they all depend, by construction, on the assumption of a low diffuse UV flux.

\section{Ackowledgements:}

I would like to thank the Conference Organizers for the invitation and financial support, and the Max-Planck-Institut für Kernphysik for hospitality and support during my visit.

\section{References}

Abdo, A. A., et al. (Fermi Coll.) 2010, ApJ, 715, 429

Abdo, A. A., et al. (Fermi Coll.) 2010b, ApJ, 723, 1082

Acciari, V. A., et al. (VERITAS Coll.) 2009, ApJ, 695, 1370

Aharonian, F. A., et al. (HEGRA Coll.), 1999, A\&A, 350, 757

Aharonian, F. 2001, Proceedings 27th ICRC (Hamburg), Invited, Rapporteur, and Highlight Papers, 250

Aharonian, F. A., et al. (HEGRA Coll.), 2001, A\&A, 366, 62

Aharonian, F. A., et al. (HEGRA Coll.), 2003, A\& $A, 403,523$

Aharonian, F. A., et al. (H. E. S. S. Coll.) 2006, Nature, 440, 1018

Aharonian, F. A., et al. (H. E. S. S. Coll.) 2007, A\&A, 473, 25

Aharonian, F. A., et al. (H. E. S. S. Coll.) 2007b, A\&SA, 475, L9

Aharonian, F. A., Khangulyan, D., \& Costamante, L. 2008, MNRAS, 387, 1206

Albert, J., et al. (MAGIC Coll.) 2008, Science, 320, 1752

Costamante, L., et al. 2004, NewAR, 48, 469

Costamante, L., 2007, ApESSS, 309, 487

Dominguez, A. et al. 2011, MNRAS, 410, 2556

Dwek, E. \& Krennrich, F., 2005, ApJ, 618, 657

Franceschini, A., et al. 2008, A\&SA, 487, 837

Gilmore, R. C., et al. 2011, MNRAS, submitted (arXiv:1104.0671)

Hauser, M. G. \& Dwek, E. 2001, ARAA, 39, 249

Hinton, J. A. \& Hofmann, W., 2009, ARAA, 47, 523

Katarzynski, K., et al. 2006, MNRAS, 368, L52

Kneiske, T. M. \& Dole, H. 2010, A\& A, 515, A19

Lefa, E., Rieger, F. M., \& Aharonian, F., 2011, ApJ, 740, 64

Madau, P. \& Silk, J. 2005, MNRAS, 359, L37

Mazin, D. \& Raue, M. 2007, A\&A 471, 439

Reimer, A., 2007, ApJ, 665, 1023

Santos, M. R. et al., 2002, MNRAS, 336, 1082

Saugé, L. \& Henri, G. 2004, ApJ 616, 136

Stecker, F. W., Baring, M. G., \& Summerlin, E. J. 2007, ApJ, 667, L29

Tavecchio, F., et al. 2009, MNRAS, 399L, 59

\section{Discussion}

Mattila: Does MAGIC give additional (or better) constraints to the EBL at optical wavelengths, where it should be more sensitive than HESS because of its lower $\gamma$-ray energy range ? (I am referring to the 2008 Science paper on 3C279).

Costamante: In this case (3C 279), not really. The problem is given by the much higher uncertainty in the spectrum. It is also caused by 1) the smaller detected band (less than half a decade, with respect to a full decade in energy for 1ES 1101-232 and the other BL Lacs), and 2) the much lower S/N. The lower $\gamma$-ray energy range probes slightly shorter wavelengths, but it also reduces the sensitivity of the $\gamma$-ray spectrum to EBL changes, with respect to the $0.2-2 \mathrm{TeV}$ range, despite the higher redshift. In the blue-UV range ("max EBL" curve in their Fig. S2), however, the values are basically 
arbitrary, and should not be considered limits at all (since a higher UV flux would also make the MAGIC spectrum softer, see Sect 2).

In summary, that observation does not provide more stringent constraints on the optical background, though the mere detection itself corroborates the idea of a low overall EBL (adopting historical fluxes for 3C 279). It is also important to bear in mind that, for this source, there is a further systematic uncertainty due to internal absorption of UV photons from the broad line region (this object has broad lines in the optical spectrum). 